Aus der Kaiserlichen chirurgischen Universitätsklinik Kyoto, Japan (Prof. H. I to).

\title{
Experimenteller Beitrag zur Knochenneubildung durch Injektion bzw. Implantation von Periost- emulsion.
}

\author{
Von Dr. T. Jokoi, Assistent der Klinik.
}

Im Jahre 1909 hatten Nakahara und Dilger in der Heidelberger chirurgischen Klinik des Professor Narath bemerkenswerte Versuche mit Periostemulsion angestellt, um auf einfache Weise Knochenneubildung zu erzeugen. Sie entnahmen das Periost jungen Tieren und zerschnitten es mit der Schere und dem Skalpell in ganz feine Stückchen. Diese Stückchen injizierten sie mit physiologischer Kochsalzlösung und Blut vermengt in die Muskulatur bzw. unter die Haut. Unter 7 Fällen hatten sie in 3 schöne Erfolge. Nach ihnen kommt es vor allem darauf an, das Periost jungen Tieren zu entnehmen, dieses möglichst vor mechanischen Insulten $\mathrm{zu}$ bewahren und schließlich in möglichst großer Menge zu injizieren.

$\mathrm{S}$ asaki setzte in derselben Klinik die Versuche weiter fort. Nachdem er sich überzeugt hatte, daß die Methode bei subkutaner Injektion brauchbare Dauerresultate lieferte, erzeugte er künstliche Pseudarthrosen und studierte daran die Heilungsvorgänge nach der Injektion von Periostemulsion.

Tsunoda überpflanzte möglichst fein zerzupfte Periostfetzen. Unter 40 Fällen fand sich nur 6 mal eine intramuskuläre Knorpel- und Knochenbildung, die sich innerhalb zwei Wochen nach der Transplantation in den zwischen den Muskelbündeln liegenden Teilen entwickelt hatte.

Es wird heutzutage niemand wohl daran zweifeln, daß auch unabhängig vom Knochen durch völlig abgetrennte und verlagerte Perioststückchen Knochen neugebildet werden kann. 
Immerhin ist zu beachten, daß bei den Versuchen von $\mathrm{Naka}$ hara und Dilger 43 Proz. und bei denen von Tsunoda nur 15 Proz. ein positives Resultat ergeben haben. Es interessierte mich zu untersuchen, unter welchen Bedingungen dasselbe wohl zustande kommt.

Eigene Versuche.

Meine Versuche wurden meist an Kaninchen, selten an jungen Hunden angestellt. Wie üblich wurde das Periost fast immer der Tibia der Versuchstiere entnommen und mit scharfen Instrumenten in kleine Stückchen zerschnitten. Die so gewonnenen Perioststückchen wurden sofort subkutan oder intramuskulär in den Oberarm oder Oberschenkel implantiert bzw. injiziert. Nach verschieden langen Zeiträumen wurden die betreffenden Teile herausgeschnitten und in Flemmingschem Säuregemisch oder Müllerscher Flüssigkeit fixiert. Die Schnitte färbte ich mit Hämatoxylin-Eosin, Safranin und Karbolfuchsin, zuweilen nach der van Giesonschen oder Weigertschen Methode.

I. Implantation von Perioststückchen auf dasselbe Tier.

In dieser Versuchsreihe wurden die Perioststückchen verdünnt mit o,85 proz. Kochsalzlösung in die Weichteile implantiert bzw. injiziert. Unter den 13 Versuchen dieser Art gingen 2 Kaninchen am nächsten Tage zugrunde, und ein drittes bekam am 5. Tage Eiterung an der operierten Stelle. Von den übrigbleibenden Io Versuchen wurden bei 4 die implantierten bzw. injizierten Stellen 5 bis 25 Tage später exstirpiert. Mikroskopisch kann man an denselben weder Knorpel- noch Knochenwachstum nachweisen. Freilich bleibt die Vitalität der Perioststückchen meistens erhalten, indem sie geschrumpft oder zusammengerollt sind und zwischen den Bindegewebsfasern entweder vereinzelt oder gehäuft sich finden. Hie und da nimmt man mit Hämatoxylin stark gefärbte verkalkte Teile wahr. Die protoplasmareichen Osteoblasten mit ovalen oder rundlichen Kernen kommen bald an der inneren Seite der Perioststückchen, bald zwischen den Bindegewebsfasern vor. Sonst findet man nicht selten kleine Blutungsherde, schwache Leukocytenemigration und spärliche 
Riesenzellen, niemals aber neugebildete Kapiliaren. An den nach der Weigertschen Methode gefärbten Schnitten ist eine breite Zone der feinen, gut gefärbten und dicht aneinander liegenden elastischen Fasern in den Perioststückchen vorhanden.

Bei weiteren 6 Fällen wurde die Exstirpation der implantierten bzw. injizierten Stellen 15 bis 70 Tage später vorgenommen, in welchen man eine mehr oder weniger starke Knochenneubildung feststellen konnte. Es sei mir gestattet, über die Versuche kurz zu berichten.

Versuch I. Ausgewachsenes Kaninchen. Subkutane Implantation. Heilung per primam. 40 Tage später wird ein platter, bohnengroßer, knorpel- bis knochenharter Knoten aus der Implantationsstelle exstirpiert.

Mikroskopisch finden sich im Knoten einige mohnsamen- bis reiskorngroße Knochenstücke, welche mit Safranin oder Fuchsin schön gefärbt werden und von einer Osteoblastenschicht oder einem Keimgewebe umgrenzt sind. Zwischen dem neugebildeten Knochen und dem Keimgewebe findet man ein osteoides und chondroides Gewebe, daneben sind zahlreiche neugebildete Kapillaren vorhanden. In relativ größeren Knochenstücken sind Haverssche Kanäle sichtbar, welche von Osteoblasten ausgekleidet sind und kleine Gefäße in sich enthalten. Die im allgemeinen relativ großen Knochenkörperchen in den zackigen Knochenhöhlen werden desto kleiner, je mehr man sich dem Zentrum der Knochenstücke nähert. An einzelnen Stellen findet man Granulationsgewebe zwischen den Bindegewebsfasern, worin Leukocyten, Kambiumzellen, große etwas schwach gefärbte Osteoblasten und fettig degenerierte oder bereits molekulär zerfallene Riesenzellen verstreut liegen. Die feinen elastischen $\mathrm{Fa}$ sern in den implantierten Perioststückchen sind gut erhalten und liegen dicht aneinander.

Versuch 2. Ausgewachsenes Kaninchen. Intramuskuläre Implantation. Fast reaktionslose Heilung. 56 Tage später findet man man an der Implantationsstelle einen $1,5 \mathrm{~cm}$ langen Strang, einem Gefäße entlang liegend. Nirgends ist Zeichen der Blutung nachweisbar.

Mikroskopisch stimmt der Befund im großen und ganzen mit dem des ersten Versuches überein. Vor allem sind Knochenlamellen deutlich zu sehen. Ferner konstatiert man mit Hämatoxylin bläulich gefärbte, verkalkte Knorpelteile. Endlich ist hier kein Granulationsgewebe aufgetreten.

Versuch 3. Anderthalb Monat altes Kaninchen. Subkutane Implantation. Heilung per primam. Ein erbsengroßer, knorpel- bis knochenharter Knoten wird I5 Tage später exstirpiert. 
Mikroskopisch sieht man mehrere hirsekorngroße Knochenstücke, von stark proliferierendem Keimgewebe umfaßt. In letzteren findet sich ein knorpeliges und osteoides Gewebe, dessen Grundsubstanz ein schönes homogenes Aussehen zeigt und aus dem Protoplasma der Osteoblasten entstanden zu sein scheint. Um das Gewebe herum begegnet man zahlreichen neugebildeten Kapillaren, welche in die Haversschen Rinnen und Kanäle hineintreten. Zwischen den Bindegewebsfasern nimmt man hier und da kleine Anhäufungen teils von Kambium- und Riesenzellen, teils von Rund- und Bindegewebszellen wahr. Verkalkte Teile und elastische Fasern sind hier gut erkennbar.

Versuch 4. Junges Kaninchen. Subkutane Injektion. Ganz reaktionslose Heilung. 27 Tage später wird ein über erbsengroßes subkutan liegendes, knochenhartes Plättchen exstirpiert, welches schon am I 5. Tage nach der Injektion sich deutlich fühlen ließ.

Mikroskopisch findet man miliargroße, in lebhafter Proliferation begriffene Knochenstücke und osteoides Gewebe, von Osteoblasten oder Keimgewebe umrahmt, mit mehreren neugebildeten Kapillaren in der Umgebung. An einigen Stellen findet sich ein aus großen, stark gefärbten Osteoblasten bestehendes Keimgewebe, dessen Zentrum etwas homogen erscheint und osteoides Aussehen hat. Die Knochenkörperchen sind meist sehr groß, und ihr Protoplasma geht ohne scharfe Grenze in die Grundsubstanz des Knochens über. Die Haversschen Kanäle sind noch nicht aufgetreten. Hier und da sieht man Granulationsgewebe, welches aus Kambium- und Rundzellen entstanden ist. Die zufällig mitinjizierten Knochensplitter sind der Resorption anheimgefallen, unter Bildung von Lakunen und Riesenzellen und von einer dünnen Bindegewebsschicht umgeben. Ihre Knochenkörperchenhöhlen sind meistens hell, und die Knochenzellen scheinen bereits zugrunde gegangen zu sein.

Versuch 5. Junges Kaninchen. Subkutane Implantation. Am nächsten Tage konstatiert man eine leichte Anschwellung an der Implantationsstelle, welche bald danach verschwindet. 25 Tage später wird ein linsengroßer, knorpelharter Knoten exstirpiert.

Mikroskopisch findet man mehrere lebhaft wuchernde, nierenglomerulusgroße, ovale oder rundliche Knochenstiicke und osteoides Gewebe, von einer einfachen Osteoblastenschicht oder einem Keimgewebe umschlossen. Die Knochenkörperchen sind hier ebenso beschaffen, wie im vorigen Versuche, und die Haversschen Kanäle und Rinnen sind nur andeutungsweise aufgetreten. Hier und da sind Leukocyten zusammengehäuft. Man nimmt wenige verkalkte Teile wahr. Riesenzellen sind nicht vorhanden. Sehr zahlreiche neugebildete Kapillaren sind hauptsächlich in der Nähe von Keimgeweben sichtbar.

V e r s u c h 6. Junges Kaninchen. Subkutane Implantation. Heilung ganz reaktionslos. 30 Tage post operationem fühlt man einen 
bohnengroßen, knochenharten Knoten unter der Haut, der danach sich nicht mehr zu vergrößern scheint. Nach weiteren 40 Tagen wird der Knoten exstirpiert.

Mikroskopisch sieht man ein reiskorngroßes, ziemlich stark wucherndes Knochengewebe, mit einer Aushöhlung, worin sich ein osteoides Gewebe findet. An der Peripherie des Knochengewebes sind Osteoblasten einschichtig aneinandergereiht, mit ganz spärlichen untermischten Riesenzellen. Der Knochen hat in seinem zentralen Teile eine lamelläre Struktur, ist mit gut ausgebildeten Haversschen Kanälen versehen und von einer dünnen Schicht faserigen Bindegewebes umzingelt. Proliferierende Keimgewebe existieren daneben ebenso reichliche, neugebildete Kapillaren und einige fettig degenerierte Kambiumzellen. Die elastischen Fasern sind im allgemeinen gut erhalten.

So konnte bei 6 unter den Io Versuchen eine mehr oder weniger starke Knochenneubildung durch die Injektion bzw. Implantation von Periostemulsion erzeugt werden. Ich kann hier nicht genug betonen, daß die lebhafte Knochenneubildung vor allem vom mechanischen Verhältnisse der implantierten bzw. injizierten Perioststückchen zu den umgebenden Geweben abhängt, wie Ollier seinerzeit darauf aufmerksam machte. Sind nämlich die Perioststückchen vollständig entfaltet, ohne geschrumpft oder zusammengerollt zu sein, so kommt die ganze Fläche der Kambiumschicht derselben mit den Geweben an den Implantations- bzw. Injektionsstellen in die innigste Berührung und wird sehr gut genährt. Je günstiger dieses mechanische Verhältnis sich gestaltet, desto stärker wird die osteoblastische Tätigkeit der Kambiumzellen. In der Tat faníd ich bei den 4 erfolglosen Versuchen die Perioststückchen immer geschrumpft oder zusammengerollt. So wäre an eine voluminöse Knochenneubildung überhaupt nicht zu denken, wenn man Perioststückchen etwa in ein sehr gefäßarmes Gewebe, wie Narbengewebe, implantieren würde. Nakahara und Dilger exstirpierten die entstandenen Knoten schon nach I5, I6 und 20 und Sasaki nach 40 Tagen. Um das weitere Schicksal des neugebildeten Knochens zu studieren, wartete ich in einem meiner Versuche mit der Exstirpation bis zum 70. Tage nach der Implantation von Periostemulsion, und konstatierte, daß die neugebildeten Knochenstücke gar nicht resorbiert waren, eher die Tendenz hatten, sich noch weiter auszubreiten. 
II. Implantation von Perioststückchen a uf andere artgleiche Tiere.

Hier stellte ich ro Versuche an 8 Kaninchen und 2 jungen Hunden an. Die beiden Hündchen waren desselben Wurfs. Von ihnen wurden 17 bzw. 32 Tage post operationem derbe bohnenbzw. zeigefingerkuppengroße Knoten herausgeschnitten und in die Müllersche Flüssigkeit eingelegt. Mikroskopisch kann man keine Knochenneubildung nachweisen. Hier und da findet man nur mitimplantierte Knochensplitter, die der lakunären Resorption anheimgefallen sind. In den zackigen Knochenhöhlen derselben fehlen meistens Knochenzellen und die Knochengrundsubstanz ist teilweise aufgefasert. Die implantierten Perioststückchen sind zusammengerollt, in einer bindegewebigen Hülle eingeschlossen und behalten ihre Vitalität zum größten Teil bei. Ferner findet sich Granulationsgewebe verstreut, welches aus Rundzellen, fettig degenerierten Osteoblasten, Bindegewebszellen usw. besteht.

Bei den 8 Kaninchen wurde das Periost zur Implantation bald einem anderen jungen Kaninchen desselben Wurfes entnommen, bald einem nicht blutverwandten. Von ihnen ging eines am 2. Tage zugrunde und ein anderes bekam im weiteren Verlaufe Eiterung an der Implantationsstelle. Unter den übrigen 6 konnte man nur an folgenden 2 Versuchen Knochenneubildung feststellen:

Versuch I. Junges Kaninchen. Subkutane Implantation von den einem anderen jungen Kaninchen desselben Wurfes entnommenen Perioststückchen. Heilung per primam. I3 Tage post operationem wird ein linsengroßer knorpelharter Knoten exstirpiert.

Mikroskopisch sieht man sehr kleine, in lebhafter Proliferation begriffene Knochenstücke, die mit den nicht ossifizierenden, geschrumpften Perioststückchen zusammen von einer bindegewebigen Hïlle umschlossen sind. Daneben existieren Granulationsgewebe, welche aus Kambium- und Riesenzellen, roten und weißen Blutkörperchen usw. bestehen. An einzelnen Stellen finden sich mitimplantierte Knochensplitter, welche resorbiert werden unter Bildung von Lakunen und zahlreichen großen Riesenzellen. Sie sind mit einigen Kambiumund Rundzellen zusammen von der Umgebung durch eine Bindegewebsschicht abgegrenzt. Die Osteoblasten sind um die neugebildeten Knochenstiicke herum regelmäßig angereiht, keine Riesenzellen darin untermischt. Die Knochenzellen, sowie die Grund- 
substanz der neugebildeten Knochenstücke und das Keimgewebe sind hier ebenso beschaffen, wie beim Versuch 4 der autoplastischen Versuchsreihe.

Versuch 2. Junges Kaninchen. Subkutane Injektion von den einem anderen, nicht blutverwandten Kaninchen entnommenen Perioststückchen. Heilung per primam. 30 Tage post operationem wird ein linsengroßer derber Knoten exstirpiert.

Mikroskopisch findet man nur zwei Knochenstücke auf, welche von schwach wucherndem Keimgewebe und einschichtigen Osteoblasten umrahmt sind. Das eine von ihnen ist mohnsamen- und das andere nierenglomerulusgroß. Daneben finden sich nichtossifizierende, zusammengerollte Perioststückchen. Ferner sieht man sehr kleine Blutungsherde, die kreuz und quer von Bindegewebsfasern durchzogen sind. Nirgends sind Riesenzellen wahrnehmbar, hier und da nur einige ausgewanderte Leukocyten. Die Haversschen Kanäle und Rinnen mit Kapillaren darin sind in den neugebildeten Knochenstücken aufgetreten. Die elastischen Fasern sind gut erhalten, und die fettig degenerierten Kambiumzellen liegen verstreut.

Aus den Ergebnissen der obigen 2 Versuche darf man wohl schließen, daß Knochenneubildung durch homoioplastische Implantation von Perioststückchen hervorgerufen werden kann. wenn auch nicht so häufig und nicht so stark, wie durch die autoplastische.

\section{Implantation von Perioststückchen auf art- fremde Tiere.}

Hierbei wurden die jungen Hunden entnommenen Perioststückchen zweimal unter die Haut von ausgewachsenen Kaninchen implantiert und ebensoviel mal umgekehrt. 20 bis 40 Tage später wurden die Implantationsstellen samt der Umgebung herausgeschnitten und in Flemmingschem Säuregemisch fixiert.

Mikroskopisch kann man keine Knochenneubildung nachweisen. Die Perioststiickchen sind von Bindegewebsfasern umschlossen und von Rundzellen infiltriert. Die Kambiumzellen sind fettig degeneriert oder zerklüftet. Zwischen den Perioststückchen finden sich Osteoblasten entweder vereinzelt oder gehäuft, die in ihren Zelleibern Vakuolenbildungen zeigen und meistens fettig degeneriert, zuweilen molekulär zerfallen sind. Hier und da kommen Riesenzellen vor. Die elastischen Fasern sind teilweise noch gut erhalten. Von Neubildung der Kapillaren und Verkalkung der Weichteile ist keine Spur vorhanden. 
$\mathrm{DaB}$ das Resultat dieser Versuchsreihe ein negatives war, ist nach unseren modernen serologischen Kenntnissen kein befremdendes. Die Gründe dafür hat Schöne auf dem vorletzten Chirurgenkongre $\beta$ angegeben.

IV. Injektionvon Periostemulsion und frischem Blut.

In dieser Versuchsreihe wurden Perioststückchen mit frischem Blute desselben Tieres subkutan injiziert, und zwar 2 mal autoplastisch und I mal homoioplastisch. Das injizierte Blut wurde jedesmal fast vollständig resorbiert, und in der Umgebung der implantierten Perioststückchen fand sich eine mehr oder weniger starke Auswanderung der Leukocyten. Diese Auswanderung war bei einem der autoplastischen Fälle sehr auffallend, das Versuchstier ging leider am 5. Tage nach der Injektion zugrunde.

Im andern der autoplastischen Fälle exstirpierte ich 30 Tage später einen erbsengroßen, knorpel- bis knochenharten Knoten.

Mikroskopisch findet man einige Knochenstücke und osteoides Gewebe. In der Nähe dieser Knochenstücke liegen geschrumpfte oder zusammengerollte Periostfetzen, welche zur Knochenneubildung nicht beigetragen haben. Etwas entfernt davon sind geringe Blutmassen nachweisbar, deren einer Teil bereits organisiert ist, ohne jedoch an der Knochenneubildung Anteil zu nehmen. Keimgewebe, Kambium. zellen, Haverssche Kanäle usw. sind bei diesem Versuche ebenso beschaffen wie beim Versuch I der autoplastischen Versuchsreihe, nur mit dem Unterschiede, daß die Verkalkungsvorgänge hier etwas stärker aufgetreten sind als dort. Riesenzellen kommen nirgends vor.

Beim homöoplastischen Fall exstirpierte ich ein kleines Klümpchen nach 35 Tagen. Das Resultat ist negativ, indem mikroskopisch weder Knochen noch osteoides Gewebe aufgefunden werden kann. Von der mitinjizierten Blutmasse ist fast keine Spur erkennbar. Die Perioststückchen sind von Bindegewebsfasern umschlossen. Daneben nimmt man wenige degenerierte Kambiumzellen, einige verkalkte Teile und Rundzelleninfiltrationen wahr.

Nakahara und Dilger gingen bei ihren Experimenten von den günstigen Resultaten aus, welche Bier bei der Behandlung von Pseudarthrosen durch die Injektion von Blut zwischen die beiden Bruchenden erhielt. Nach meinen obigen Versuchen scheint jedoch das mitinjizierte Blut auf die osteoblastische Tätigkeit der überpflanzten Perioststückchen keinen besonders günstigen Einfluß zu haben. Hätte ich freilich, wie 
Experimenteller Beitrag zur Knochenneubildung durch Injektion usw. 44I

Pochhammer, den Blutkuchen mitimplantiert, so dürfte ich ein günstigeres Resultat bekommen haben.

\section{Injektion von Periostemulsion und Fibrin.}

Bei dieser Versuchsreihe injizierte ich an 2 Kaninchen die Periostemulsion mit Io bis 20 Tropfen von der I proz. Fibrinlösung.

Versuch I. Junges Kaninchen. Autoplastische intramuskuläre Injektion. Nach 15 Tagen wird ein bohnengroßer harter Knoten exstirpiert.

Mikroskopisch sieht man einige gewundene Knochenspangen mit Knorpel und osteoidem Gewebe zwischen den Muskelbündeln. Sie sind überall von außerordentlich stark proliferierendem Keimgewebe umgrenzt und haben in ihrer Umgebung mehrere neugebildete Kapillaren. Knochenkörperchen, Grundsubstanz und Haverssche Kanäle sind hier ebenso beschaffen, wie beim Versuch 3 der autoplastischen Versuchsreihe. Hier und da finden sich Perioststückchen, welche an der Knochenneubildung keinen Teil genommen haben, ferner zufällig mitinjizierte, meistens von Bindegewebsfasern umschlossene Knochensplitter, welche der lakunären Resorption anheimgefallen sind. Degenerierte oder atrophierte Muskelfasern sind in der Nähe der letzteren vorhanden.

Versuch 2. Ausgewachsenes Kaninchen. Homoioplastische subkutane Injektion. Am 26. Tage nach der Injektion wird ein pfenniggroßes knochenhartes Stück exstirpiert.

Mikroskopisch findet man einige längliche Knochenstücke und osteoides Gewebe. Die Proliferation der Gewebe ist hier bei weitem stärker, als bei den Versuchen der homoioplastischen Versuchsreihe. Die Osteoblasten sind regelmäßig um die neugebildeten Knochenstiicke herum angereiht. Dicht daneben sieht man geschrumpfte Perioststückchen, welche an der Knochenneubildung nicht teilgenommen haben und von Rundzellen infiltriert sind. Gefäße und Haverssche Kanäle sind hier fast ebenso beschaffen, wie beim vorigen Versuche. Mitinjizierte Knochensplitter sind der lakunären Resorption anheimgefallen.

Nach den Ergebnissen der obigen Versuche scheint das Fibrin die Kambiumzellen der überpflanzten Perioststückchen zur gesteigerten Knochenneubildung anzuregen, wie Bergel durch subperiostale Injektionen von Fibrinemulsion starke kallöse Knochenverdickungen hervorrufen konnte. 
VI. Injektion von abgeschabter Kambiumschicht.

Die Injektion von abgeschabter Kambiumschicht ergab in den Versuchen von Nakahara und Dilger, Sasaki und Tsunoda immer ein negatives Resultat. Poch hammer konnte hingegen schon nach 10-I4 Tagen im Röntgenbilde an der Stelle der Überpflanzung einen kleinen, meist punktförmigen Knochenschatten konstatieren und in zwei Fällen später auch histologisch Knorpel- und Knochenbildung nachweisen. Um entscheiden zu können, wer recht hat, wiederholte ich selbst die obigen Experimente.

Ich strich die innere Schicht eines von der Tibia abpräparierten Periostlappens sowie die entblößte Knochenoberfläche mehrmals ab. Die so gewonnene, mit physiologischer Kochsalzlösung verdünnte Masse wurde 2 mal subkutan und I mal intramuskulär in den Oberschenkel injiziert.

8, 20 bzw. 35 Tage später wurden die injizierten Stellen exstirpiert und histologisch untersucht. Die Ergebnisse waren immer negativ, indem die injizierte Masse fast vollständig resorbiert worden war. Man fand nur wenige ausgewanderte Leukocyten, spärliches Granulationsgewebe und leicht degenerierte Muskelfasern.

Somit scheint den auf mechanischem Wege ganz isolierten Osteoblasten die knochenbildende Tätigkeit nicht eigen zu sein.

Fasse ich die Resultate vorstehender Versuche noch einmal kurz zusammen, so finde ich:

I. Durch die autoplastische Implantation bzw. Injektion von Periostemulsion konnte ich bei 6 unter Io Versuchen eine mehr oder weniger starke Knochenneubildung erzeugen.

2. Die lebhafte Knochenneubildung hängt dabei vor allem vom mechanischen Verhältnisse der implantierten bzw. injizierten Perioststückchen zu den umgebenden Geweben ab. Bei den erfolglosen Versuchen sind dieselben immer geschrumpft oder zusammengerollt.

3. In einem der Versuche konstatierte ich, daß die neugebildeten Knochenstücke bis zum 70. Tage nach der Implan: tation gar nicht resorbiert waren, eher die Tendenz hatten sich noch weiter auszubreiten. 
4. Auch durch homoioplastische Implantation bzw. Injektion von Periostemulsion kann Knochenneubildung hervorgerufen werden, wenn auch nicht so häufig und nicht so stark wie durch die autoplastische.

5. Durch heteroplastische Implantation bzw. Injektion von Periostemulsion können keine Knochenstücke neugebildet werden.

6. Das mitinjizierte frische Blut desselben Tieres scheint auf die osteoblastische Tätigkeit der überpflanzten Perioststückchen keinen besonders günstigen Einfluß zu haben.

7. Das mitinjizierte Fibrin scheint hingegen die Kambiumzellen der überpflanzten Perioststückchen zur gesteigerten Knochenneubildung anzuregen.

8. Durch die Injektion von abgeschabter Kambiumschicht ist man nicht imstande, Knochenneubildung zu erzeugen.

9. In allen Versuchen fallen zufällig mitinjizierte Knochenteilchen fast immer der lakunären Resorption anheim.

\section{Literaturverzeichnis.}

I. B e rge 1, S., Callusbildung durch Fibrin. Arch. f. klin. Chir. I9Ir, Bd. 93 .

2. Bier, August, Die Bedeutung des Blutergusses für die Heilung des Knochenbruches. Heilung von Pseudarthrosen und von verspäteter Callusbildung durch Bluteinspritzung. Deutsche med. Klinik I905, Bd. I,

3. Nakahara, T., u. Dilger, A., Subkutane und intramuskuläre Knochenneubildungen durch Injektion bzw. Implantation von Periostemulsion. Beitr. z. klin. Chir. I909, Bd. 63.

4. Olli er, Léopold, De la production artificielle des os au moyen de la transplantation du périoste et des greffes osseuses. Comptes rendus des séances et mémoires de la société de biologie, tome 5 , série 2 , année 1858 .

5. Pochhammer, Conrad, Uber die Entstehung periostaler Callusbildungen und die künstliche Calluserzeugung an Tieren und beim Menschen. Arch. f. klin. Chir. Igr I, Bd. 94.

6. Sasaki, J., Über die Behandlung der Pseudarthrosen durch Injektion von Periostemulsion. Deutsche Zeitschr. f. Chir. I9II, Bd. Io9.

7. Schön e, G., Transplantationsversuche mit artgleichen und artfremden Geweben. Verhandl. d. Deutsch. Gesellsch. f. Chir., 40. Kongreß, Berlin IgI I.

8. Tsunoda, T., Experimentelle Studie zur Frage der Knochenbildung aus verlagerten Periostosteoblasten. Virchows Arch. I9I0, Bd. 200. 